\title{
Impact of single versus double transseptal puncture on outcome and complications in pulmonary vein isolation procedures
}

\author{
Annina Stauber ${ }^{1}$, Jelena Kornej ${ }^{1}$, Alireeza Sepehri Shamloo ${ }^{1}$, Boris Dinov ${ }^{1}$, \\ Justinas Bacevicius ${ }^{1}$, Nikolaos Dagres ${ }^{1}$, Andreas Bollmann ${ }^{1}$, \\ Gerhard Hindricks ${ }^{1,2}$, Philipp Sommer ${ }^{1,2,3}$ \\ ${ }^{1}$ Department of Electrophysiology, Heart Center University Leipzig, Germany \\ ${ }^{2}$ Leipzig Heart Institute, Leipzig, Germany \\ ${ }^{3}$ Clinic of Electrophysiology, Heart and Diabetes Center NRW, University Hospital \\ of Ruhr-University Bochum, Bad Oeynhausen, Germany
}

\begin{abstract}
Background: The aim of the current study was to analyze the impact of single versus double transseptal puncture (TSP) for atrial fibrillation (AF) ablation.

Methods: Consecutive patients undergoing $A F$ ablation were prospectively included in the $A F$ ablation registry and were analyzed according to single versus double TSP.

Results: A total of 478 patients (female 35\%, persistent $A F$ 67\%) undergoing AF ablation between 01/2014 and 09/2014 were included. Single TSP was performed in 202 (42\%) patients, double TSP in $276(58 \%)$ patients. Age, gender, body mass index, $\mathrm{CHA}_{2} D S_{2}-V A S c$ score, left ventricular ejection fraction and operator experience (experienced operator defined as $\geq 5$ years of experience in invasive electrophysiology) were equally distributed between the two groups. Repeat procedures (re-dos) were more frequently performed using single TSP access $(p<0.001)$. Left atrial $(L A)$ diameter was larger in patients with double $\operatorname{TSP}(p=0.001)$. Procedure duration in single TSP was identical to double TSP procedures $(p=0.823)$. Radiation duration was similar between the two groups $(p=0.217)$. There were $49(10 \%)$ patients with complications after catheter ablation. There were no differences between complication rates and TSP type ( $p=0.555)$. Similarly, recurrence rates were comparable between both TSP groups ( $p=0.788$ ).

Conclusions: There was no clear benefit of single or double TSP in AF ablation. (Cardiol J 2021; 28, 5: 671-677)
\end{abstract}

Key words: atrial fibrillation, catheter ablation, pulmonary vein isolation, transseptal puncture, complications

\section{Introduction}

Transseptal puncture (TSP) is one of the most challenging steps in catheter ablation of atrial fibrillation (AF). It is a critical moment because of the potential risk of aortic puncture or puncture of the pericardial space. While single TSP reduces the risk associated with the puncture, double transseptal access simplifies the procedure in terms of immediate visualization of signals in the pulmonary vein, as well as avoidance of multiple changes of ablation and multipolar catheter through the single sheath.

There are three options for transseptal access. First, the single transseptal approach strategy. Second, the single-puncture-double-transseptal approach with one puncture being performed and the second sheath/catheter being advanced in the

Address for correspondence: Annina Stauber, MD, Cardiology Cologne University Clinic, Kerpener Strasse 62, 50937 Cologne, Germany, tel: 0049221478 32396, fax: 0049221 47832397, e-mail: annina.stauber@gmx.net

Received: 8.10.2019 Accepted: 1.03.2020 Early publication date: 18.03 .2020

This article is available in open access under Creative Common Attribution-Non-Commercial-No Derivatives 4.0 International (CC BY-NC-ND 4.0) license, allowing to download articles and share them with others as long as they credit the authors and the publisher, but without permission to change them in any way or use them commercially. 
left atrium (LA) beneath the first access site [1] Thirdly, there is the option of double-puncture-double-transseptal access. Despite the great practical relevance, the impact of this decision by the operator is yet unclear - therefore, the aim of this study was to investigate whether single or double transseptal access is superior in terms of procedure time, radiation time, complication rates and outcome.

\section{Methods}

Consecutive patients admitted for ablation of $\mathrm{AF}$ were prospectively included in the $\mathrm{AF}$ ablation registry. The Leipzig AF Ablation Registry has been approved by the Ethics Authority. Data from patients between January 2014 and September 2014 were analyzed. The patients were $\geq 18$ years old. Patients undergoing cryo-ablation and procedures with radiation-saving technology (MediGuide, Abbott, St. Paul, MN, USA) were excluded to allow an unbiased comparison of the datasets.

Baseline characteristics were analyzed, procedural aspects with a focus on procedure and fluoroscopy time, complication rates, and follow-up data are presented herein.

\section{Ablation procedure and TSP}

Indication for catheter ablation was based on the current European Society of Cardiology Guidelines [2]. Procedural steps have been described prior [3]. In brief, the patients were deeply sedated (midazolam, propofol) and received analgetics (fentanyl) as described by Kottkamp et al. [4]. Placement of the diagnostic right ventricular apex and coronary sinus catheter was performed via left femoral venous access. Invasive arterial monitoring was performed via left femoral artery. Sheaths for TSP were placed into the right femoral vein. The decision for single versus double TSP was at the operators' discretion.

In cases of a single TSP, the guide wire was advanced into the superior vena cava. The steerable sheath (Agilis, Abbott, St. Paul, MN, USA); the curve $[S, M, L]$ of the sheath was selected on the basis of a previously performed cardiac magnetic resonance imaging $[\mathrm{CMR}]$ ) was advanced into the superior vena cava, the wire was removed and the TSP needle was inserted. In a left anterior oblique (LAO) view, the steerable sheath was withdrawn until typically 2 "jumps" were observed. The position was confirmed in a right anterior oblique view (RAO). A small amount of contrast dye was injected to prove septal tenting in LAO. Then, the puncture was performed and documented with an X-ray film. As soon as the needle was in the LA, the correct localization was confirmed by contrast dye injection and optionally by recording pressure via the needle tip. Subsequently, the steerable sheath was advanced into the LA, and the needle withdrawn. If a second TSP was planned, the same steps were performed with a non-steerable long sheath (SL 0, Abbott, St. Paul, MN, USA).

An electroanatomical reconstruction of the LA was performed by use of a three-dimensional (3D) mapping system (Carto 3, Biosense Webster, Diamond Bar, CA, USA; Ensite Velocity, Abbott, St. Paul, MN, USA), in a subgroup of these patients a fusion between the reconstructed 3D-CMR model and the electroanatomical reconstruction was done. Isolation of the pulmonary veins was confirmed by bidirectional block around the ipsilateral veins at an antral level. Linear lesions or focal ablations were added according to voltage information that was collected during sinus rhythm in all patients (Figs. 1, 2) [5].

\section{Follow-up}

Routine follow-up at the documented center included visits at 3,6, and 9 months after ablation and then every 12 months thereafter [3]. Early recurrences within 3 months were considered as a blanking period. Atrial arrhythmias $(\geq 30 \mathrm{~s})$ were defined as recurrences. Usually, an electrocardiogram (ECG) was performed on each visit. If patients complained about symptoms, intensified resting and 1-7 days Holter-ECG-monitoring was performed. Only patients with at least one Holter-ECG or implantable device (pacemaker or implantable cardioverter-defibrillator [ICD]) and a follow-up of at least 6 months were included into the recurrence analysis.

\section{Complications}

Complications were classified into three groups: pericardial effusion (PE), groin complications and cerebrovascular incidents. PE was counted if relevant effusion was detected, puncture or operation was required. Groin complications were counted if a procedure (control, injection, stenting or operation) was required. The third category was cerebrovascular incidents including stroke and transient ischemic attack (TIA).

\section{Statistical analyses}

Mean values (and standard deviation [SD]) for normally distributed data, median (and interquartile range $[\mathrm{IQR}]$ ) for skewed data and for categori- 


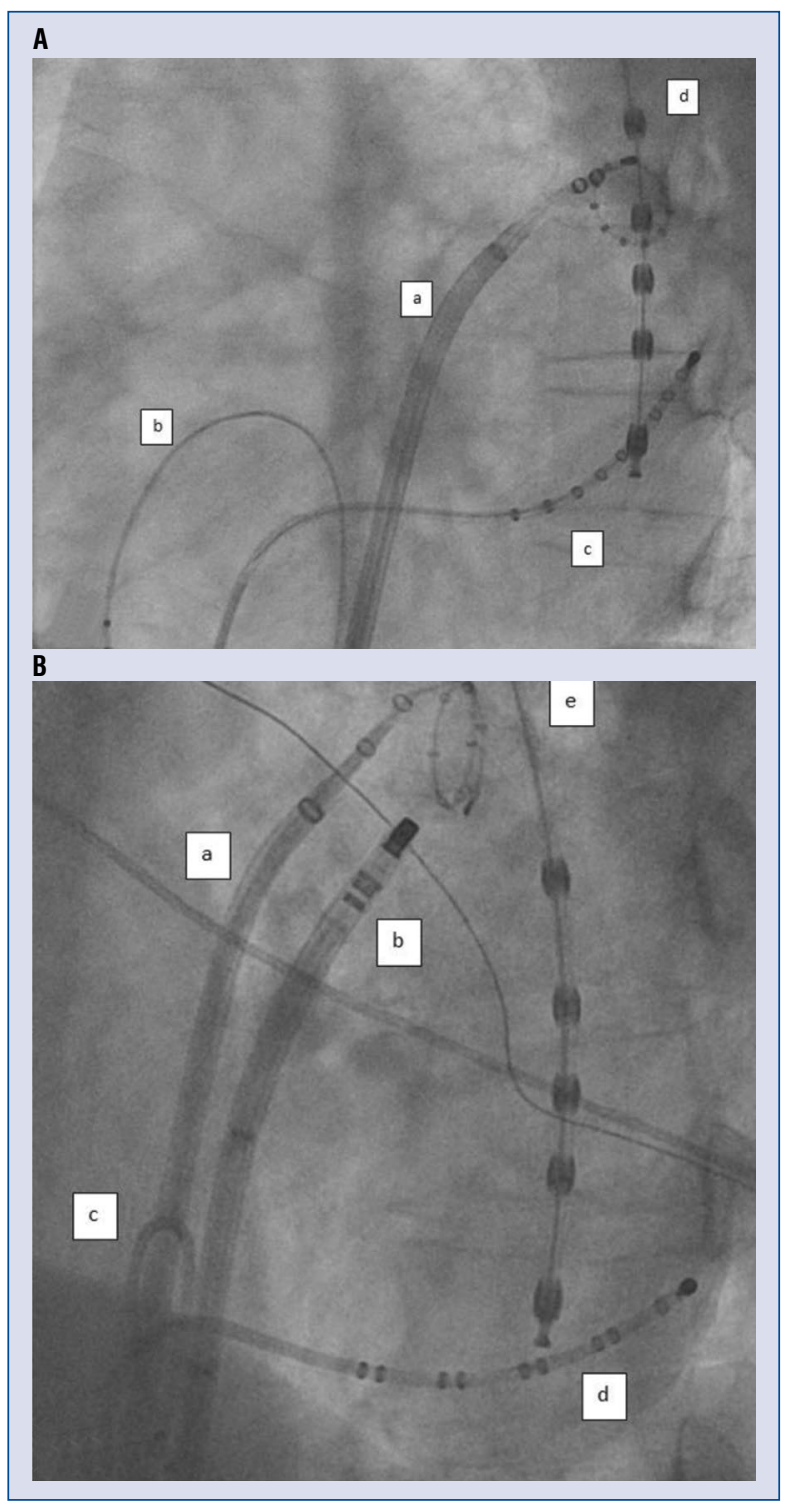

Figure 1. A. Left anterior oblique (LAO) $50^{\circ}$ view. Single transseptal puncture; a - Agilis sheath (St. Jude, Abbott, St. Paul, MN, USA) in the left atrium with a 10 polar spiral-catheter in the left superior pulmonary vein; b - diagnostic catheter in the right ventricular apex; c - diagnostic catheter in the coronary sinus; $d$ - temperature probe in esophagus; B. LAO $50^{\circ}$ view. Double transseptal puncture; a - SL0 Sheath (St. Jude, Abbott, St. Paul, MN, USA) in the left atrium with a 10 polar spiral-catheter in the left superior pulmonary vein; $b$ Agilis sheath (St. Jude, Abbott, St. Paul, MN, USA) with ablation catheter ostial of the left superior pulmonary vein; c - diagnostic catheter in the right ventricular apex; $d$ - diagnostic catheter in coronary sinus; e temperature probe in the esophagus.

cal data proportions in percentage were used. The Spearman rank method was used for correlations. The unpaired t-test and the Mann-Whitney test were used for differences in continuous variables and $\chi^{2}$ test for differences in categorical variables. Multivariable analysis (including variables with a p-value $<0.2$ found on univariable analysis) was performed to find predictors for the complications. A p-value $<0.05$ was defined as statistically significant. The statistical analyses were done with SPSS statistical software version 23 (SPSS Inc., Chicago, USA).

\section{Results}

The study included 478 patients undergoing radiofrequency $\mathrm{AF}$ catheter ablation between January and September 2014 at the Heart Center Leipzig (age $62 \pm 10$ years, 35\% females, $67 \%$ persistent AF). The median follow-up was 23 months (IQR 4-37).

Single TSP was performed in $202(42 \%)$ patients, double TSP in 276 (58\%) patients. Age, gender, body mass index and $\mathrm{CHA}_{2} \mathrm{DS}_{2}$-VASc score, left ventricular ejection fraction and operator experience (defined as $\geq 5$ years of experience in invasive electrophysiology) were equally distributed between the two groups. Repeat procedures (re-dos) were more frequently performed using single TSP access $(p<0.001)$. LA diameter was larger in patients with double TSP $(\mathrm{p}=0.001)$. Procedure duration did not differ significantly between the two groups $(\mathrm{p}=0.823)$. Radiation duration was similar for the two groups $(p=0.217)$, but the radiation dose was significantly lower in single TSP procedures $(\mathrm{p}<0.001)$. TSP type did not affect the recurrence rate $(\mathrm{p}=0.788)$ (Table 1 , Fig. 3 ).

\section{Complications}

There were $49(10 \%)$ patients with clinically relevant complications. There were $25(5.2 \%)$ patients with pericardial effusion/tamponade, $19(4.0 \%)$ with groin complications, and $5(1 \%)$ patients suffered stroke/TIA. There was no significant difference in total complication rates between single and double TSP ( $\mathrm{p}=0.555)$, however, numerically there were less $\mathrm{PE}$ (10 vs. 15), less groin complications $(\mathrm{n}=9$ vs. $\mathrm{n}=10)$ and less strokes $(n=1$ vs. $n=4)$ in the single TSP group (Table 2$)$. Univariable analysis showed no significant association between age, gender of patient, AF type, LA size, $\mathrm{CHA}_{2} \mathrm{DS}_{2}$-VASc score, re-do procedures or operator experience on the complication rate (Table 3 ).

\section{Rhythm outcomes}

During follow-up, 195 (41\%) patients received long-term monitoring with Holter-ECG or had an 


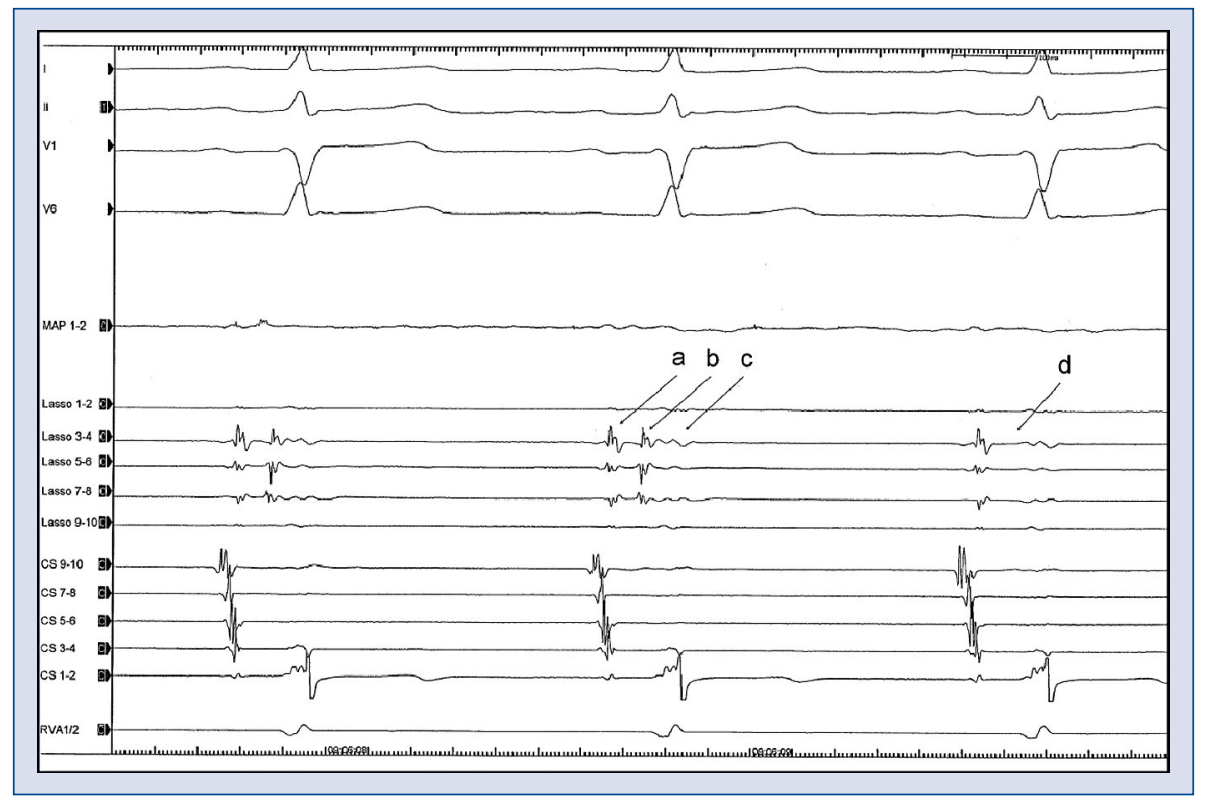

Figure 2. Electrocardiogramm during ablation. I, II, V1, V6 = Surface-electrocardiogram, MAP = ablation catheter, Lasso $1-10=10$ polar spiral-catheter in the left superior pulmonary vein: $a$ - farfield atrial signal; $b$ - pulmonary vein signal; c - farfield ventricular signal; $d$ - no pulmonary vein signal anymore; CS 1-10 - catheter in the coronary sinus; RVA - catheter in the right ventricular apex. The 10 polar spiral-catheter is placed in the left superior pulmonary vein. During ablation around the left superior pulmonary vein, the pulmonary vein signal on the spiral-catheter disappears $(b \rightarrow d)$. This means that the vein was isolated, because there was hence, no signal passing the ablation line.

Table 1. Clinical characteristics of the study population.

\begin{tabular}{|c|c|c|c|}
\hline & Single TSP $(n=202)$ & Double TSP $(n=276)$ & $\mathbf{P}$ \\
\hline Age [years] & $62(55-71)$ & $64(57-71)$ & 0.110 \\
\hline Females, & $79(39 \%)$ & $89(32 \%)$ & 0.131 \\
\hline Persistent AF & $138(68 \%)$ & $184(67 \%)$ & 0.762 \\
\hline Re-ablation & $75(37 \%)$ & $48(17 \%)$ & $<0.001$ \\
\hline BMI $\left[\mathrm{kg} / \mathrm{m}^{2}\right]$ & $28(25-32)$ & $28(26-32)$ & 0.119 \\
\hline $\mathrm{CHA}_{2} \mathrm{DS}_{2}$-VASc score & $2(1-3)$ & $2(1-3)$ & 0.339 \\
\hline LAd $[\mathrm{mm}]$ & $43(39-47)$ & $45(41-48)$ & 0.001 \\
\hline LVEF [\%] & $60(55-64)$ & $60(51-64)$ & 0.781 \\
\hline Radiation time [min] & $18.6(11.5-25.5)$ & $16.2(10.1-25.0)$ & 0.217 \\
\hline Radiation dose $\left[\mathrm{cGycm}^{2}\right]$ & $3782(1.800-7.200)$ & $6200(3.038-10.323)$ & $<0.001$ \\
\hline Procedure time [min] & $150(120-180)$ & $145(120-175)$ & 0.823 \\
\hline Experienced operator & $74 \%$ & $79 \%$ & 0.208 \\
\hline Recurrences $>6$ months* & $55(71 \%)$ & $79(69 \%)$ & 0.788 \\
\hline Complications & $20(10 \%)$ & $29(11 \%)$ & 0.555 \\
\hline
\end{tabular}

${ }^{*}$ Recurrences $>6$ months in patients with available implanted device (pacemaker, ICD, ILR) available in 193 patients (40\% of the study population). Data presented as number (\%) or median (interquartile range); AF — atrial fibrillation; BMI — body mass index; LAd — left atrial diameter; LVEF - left ventricular ejection fraction; TSP — transseptal puncture

implantable device such as pacemaker/defibrillator allowing continuous monitoring and had a followup of 6 months or more. In this subgroup, there were $55(71 \%)$ and $79(69 \%)$ with recurrences for single and double TSP, respectively $(\mathrm{p}=0.788)$ (Table 1, Fig. 3). 


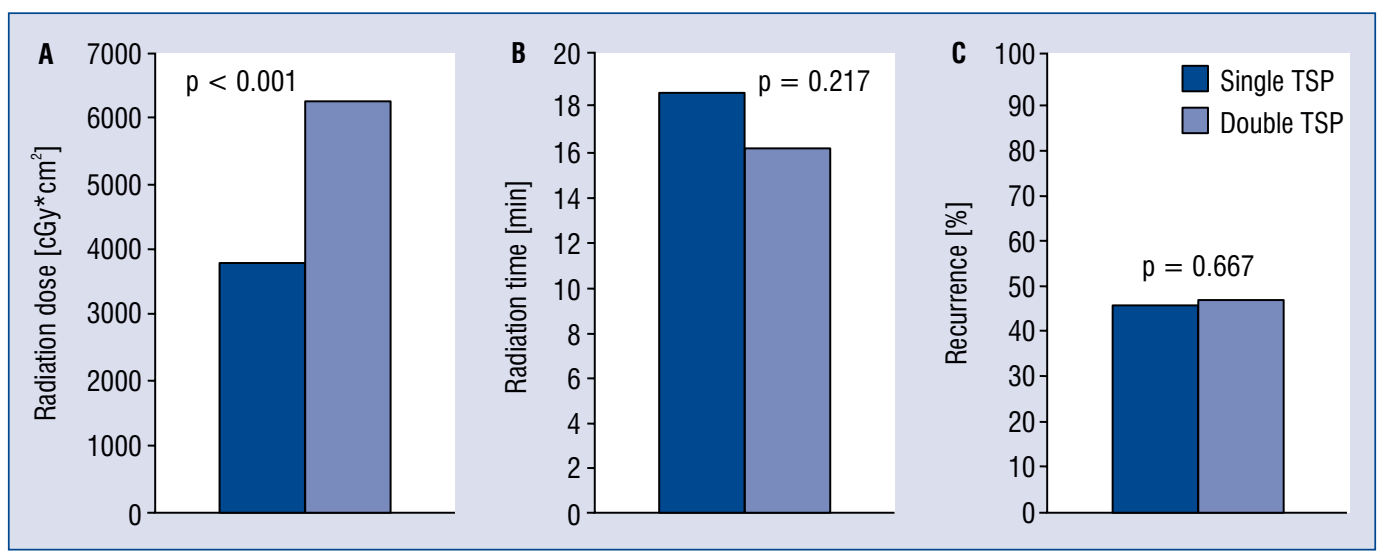

Figure 3. A. Radiation dose in single and double transseptal puncture (TSP); B. Radiation time in single and double TSP; C. Recurrence in single and double TSP.

Table 2. Complications accordingly to the transseptal puncture (TSP) type; $p=0.555$.

\begin{tabular}{lccc}
\hline & Total $(\mathbf{n}=\mathbf{4 7 8})$ & Single TSP $(\mathbf{n}=202)$ & Double TSP $(\mathbf{n}=\mathbf{2 7 6})$ \\
\hline None & $429(89.6 \%)$ & $182(89.7 \%)$ & $247(89.5 \%)$ \\
Pericardial effusion & $25(5.2 \%)$ & $10(4.9 \%)$ & $15(5.4 \%)$ \\
Groin complications & $19(4.0 \%)$ & $9(4.4 \%)$ & $10(3.6 \%)$ \\
Strokes & $5(1.0 \%)$ & $1(0.5 \%)$ & $4(1.4 \%)$ \\
\hline
\end{tabular}

Table 3. Prediction of complications.

\begin{tabular}{lcc}
\hline & \multicolumn{2}{c}{ Univariable analysis } \\
\cline { 2 - 3 } & \multicolumn{1}{c}{$\begin{array}{c}\text { Odds ratio } \\
(95 \% \mathrm{CI})\end{array}$} & $\mathrm{P}$ \\
\hline Age & $1.034(0.984-1.087)$ & 0.187 \\
Females & $2.124(0.846-5.334)$ & 0.109 \\
Persistent AF & $1.059(0.395-2.840)$ & 0.910 \\
$\mathrm{BMI}^{2} \mathrm{~kg} / \mathrm{m}^{2}$ ] & $0.957(0.868-1.055)$ & 0.376 \\
$\mathrm{CHA}_{2} \mathrm{DS}{ }_{2}$-VASc score & $1.240(0.925-1.662)$ & 0.151 \\
Re ablation of AF & $1.035(0.365-2.935)$ & 0.948 \\
Experienced operator & $1.602(0.458-5.601)$ & 0.461 \\
TSP type & $1.623(0.606-4.345)$ & 0.335 \\
Procedure time [min] & $1.006(0.997-1.016)$ & 0.171 \\
Radiation time [min] & $1.013(0.971-1.057)$ & 0.551 \\
LAd [mm] & $1.031(0.958-1.109)$ & 0.413 \\
\hline Cl- confidence interval; AF - atrial fibrillation; BMI-body mass
\end{tabular}

index; TSP — transseptal puncture; LAd — left atrial diameter

\section{Discussion}

\section{Transseptal puncture}

Despite the large and growing number of $\mathrm{AF}$ ablations and the practical relevance to the question whether single or double TSP is better, there is, according to available research, no study comparing single versus double TSP for AF ablation procedures.

The TSP is a crucial moment in the procedure of pulmonary vein isolation. Complications of TSP are puncture of the aorta and puncture of the posterior pericardial space. While in the SAFER Registry $0.9 \%$ PEs were described in all procedures [6], Haegeli et al. [7] showed, in double TSP procedures that there were $2.6 \%$ of pericardial effusions which required an intervention. Katritsis et al. [8] have shown that TSP in AF ablation procedures are associated with a higher incidence of pericardial tamponade compared to TSP in other cardiac procedures. In the present study population, the overall rate of PE was $5 \%$, but $\mathrm{PE}$ requiring an intervention was low with only $0.8 \%$. This is likely due to the large experience at the documented center.

The number of recurrences is high. However, because Holter monitoring was intensified in patients with symptoms and only those with Holter-ECG (or pacemaker/ICD) were included in the analysis, the rate of recurrences is likely estimated too high. 
Some findings are interesting and the results need further explanations: for example, the finding that the X-ray time did not differ significantly between the two groups. Possibly, the higher radiation time which is needed for the second TSP in the double TSP group was compensated by the need for fluoroscopic control of the spiral and ablation catheter during the catheter exchange. That for each TSP an X-ray film was recorded, was probably the reason for a higher radiation dose in the double TSP group.

Secondly, the finding that single TSP only was more frequently performed in re-do procedures. This can be explained by the sometimes more challenging TSP because of an induration of the interatrial septum - therefore some operators may have skipped the initially planned second TSP.

And thirdly, there were more double TSP in larger LA diameters. Probably, operators skipped the second TSP in small LA due to anticipation of negative effects of 2 transseptal sheaths in a small LA. Interestingly despite the fact that double TSP needs an additional access in the groin for a second sheath, an only statistically non-significant difference was observed in groin complication rate between the two groups.

Silent cerebral events are more frequent in single transseptal access LA ablations, compared to double tansseptal access, due to the need for exchanging catheters over a single transseptal access as described by Deneke et al. [9]. In the current study, silent cerebral events were not assessed, for instance by use of magnetic resonance imaging after ablation. Although double TSP was associated with more clinical cerebrovascular events compared to single TSP, the difference was not significant. Pathophysiologically, micro air-embolisms are most likely to be caused by catheter exchanges, while macro embolisms are usually caused by thrombi. This may explain the difference in the results.

Overall, both approaches have advantages and disadvantages. The double TSP access has the advantage that one can simultaneously monitor the electrical signals in the pulmonary veins. Thus, the operator can often stop the ablation as soon as the signals in the pulmonary veins have disappeared. Furthermore, in linear lesions it is easier to check the lines by differential pacing. A single TSP requires more experience of the operator to promptly detect the signals in the pulmonary veins. Here, the pace and ablate strategy was frequently used for verification before the ablation catheter is taken out and multipolar catheter (Lasso, Bio- sense Webster, Diamond Bar, CA, USA or Advisor, Abbott, St. Paul, MN, USA) is inserted. This is an excellent method because pacing to ensure unexcitability along ablation lines has demonstrated to improve outcomes compared with bidirectional block alone [10]. It might be expected that with continuous pulmonary vein potential monitoring in double TSP, it is possible to reduce the duration of the procedure. On the other hand, the second TSP takes time. There was an inablity to show that ultimately, the procedure time tends to be shorter with double TSP.

It should be mentioned that all double TSP were performed by double puncture. Single-puncture double-transseptal access is not performed at the documented center. However, the latter has been shown to be safe in previous studies [11].

\section{Limitations of the study}

The main limitation of the study is that the decision on whether to use single or double TSP was at the operators' discretion and not based on randomization. On the other hand, 369 (77\%) of the procedures were performed by operators who have $\geq 5$ years invasive electrophysiological experience and thus the expertise was high and equally distributed between the two groups, reducing bias. It may be that in smaller LA, single TSP was preferred due to reasons of steerabilty.

Another limitation is the lack of assessment of iatrogenic ASD (iASD) after the procedure. However, Hammerstingl et al. [12] reported that persistent iASD occurred after double access through one puncture in 8 out of $27(30 \%)$ patients. The study of Rillig et al. [13] has shown 1 out of 31 (3\%) patients have a persistent iASD 12 months after double TSP. Cryo-balloon PVI also often goes along with a persistent iASD because of the use of a 12 French sheath (FlexCath Advance, Medtronic, Minneapolis, MN, USA) [14]. This sheath is larger than Agilis and SL 0 (8 and 7 French, Abbott, St. Paul, MN, USA). Nevertheless, iatrogenic ASD has not been found to lead to an increased risk of paradoxical embolism or relevant shunting $[13,15]$.

\section{Complications}

In the present study, there was no inverse association of the operator experience and lower complication rates. This might be a result of the fact, that experienced operators performed more complex procedures. Female sex was not associated with higher complication rates as it is described in the literature [16-21]. This could be partly explained by a relatively small ablation 
cohort and a low number of complications. Neither was a higher $\mathrm{CHA}_{2} \mathrm{DS}_{2}$-VASc score associated with higher complication rate. $\mathrm{CHA}_{2} \mathrm{DS}_{2}$-VASc score and early institutional experience showed a higher complication rate in the literature [19-22]. This was also attributed to the small sample size and low complication rate.

\section{Conclusions}

There was no clear benefit of single or double TSP in AF ablation. Recurrence and complication rate did not differ significantly.

Conflict of interest: Philipp Sommer has received lecture fees and travel support from Abbott and is member of the advisory board for Abbott and Biosense Webster. Gerhard Hindricks and Nikolaos Dagres report research grants from Abbott and Boston Scientific to the institution (Heart Center Leipzig) without any personal financial benefits.

\section{References}

1. Epstein A, Plumb V, Kay G. One-puncture, double-transseptal catheterization manoeuvre in the catheter ablation of atrial fibrillation. EP Europace. 2007; 9(7): 487-489, doi: 10.1093/europace/eum070.

2. Kirchhof P, Benussi S, Kotecha D, et al. 2016 ESC Guidelines for the management of atrial fibrillation developed in collaboration with EACTS. Europace. 2016; 18(11): 1609-1678, doi: 10.1093/ europace/euw295.

3. Dagres N, Hindricks G, Kottkamp H, et al. Complications of atrial fibrillation ablation in a high-volume center in 1,000 procedures: still cause for concern? J Cardiovasc Electrophysiol. 2009; 20(9): 1014-1019, doi: 10.1111/j.1540-8167.2009.01493.x, indexed in Pubmed: 19490383.

4. Kottkamp H, Hindricks G, Eitel C, et al. Deep sedation for catheter ablation of atrial fibrillation: a prospective study in 650 consecutive patients. J Cardiovasc Electrophysiol. 2011; 22(12): 1339-1343, doi: 10.1111/j.1540-8167.2011.02120.x, indexed in Pubmed: 21692895.

5. Rolf S, Kircher S, Arya A, et al. Tailored atrial substrate modification based on low-voltage areas in catheter ablation of atrial fibrillation. Circ Arrhythm Electrophysiol. 2014; 7(5): 825-833, doi: 10.1161/CIRCEP.113.001251, indexed in Pubmed: 25151631.

6. Bollmann A, Ueberham L, Schuler E, et al. Cardiac tamponade in catheter ablation of atrial fibrillation: German-wide analysis of 21141 procedures in the Helios atrial fibrillation ablation registry (SAFER). Europace. 2018; 20(12): 1944-1951, doi: 10.1093/ europace/euy131, indexed in Pubmed: 29982554.

7. Haegeli LM, Wolber T, Ercin E, et al. Double transseptal puncture for catheter ablation of atrial fibrillation: safety of the technique and its use in the outpatient setting. Cardiol Res Pract. 2010; 2010: 295297, doi: 10.4061/2010/295297, indexed in Pubmed: 21197071.

8. Katritsis GD, Siontis GCM, Giazitzoglou E, et al. Complications of transseptal catheterization for different cardiac procedures. Int J Cardiol. 2013; 168(6): 5352-5354, doi: 10.1016/j. ijcard.2013.08.004, indexed in Pubmed: 24012276.

9. Deneke T, Nentwich K, Schmitt R, et al. Exchanging Catheters Over a Single Transseptal Sheath During Left Atrial Ablation is
Associated with a Higher Risk for Silent Cerebral Events. Indian Pacing Electrophysiol J. 2014; 14(5): 240-249, doi: 10.1016/ s0972-6292(16)30795-1, indexed in Pubmed: 25408564.

10. Steven D, Sultan A, Reddy V, et al. Benefit of pulmonary vein isolation guided by loss of pace capture on the ablation line: results from a prospective 2-center randomized trial. J Am Coll Cardiol. 2013; 62(1): 44-50, doi: 10.1016/j.jacc.2013.03.059, indexed in Pubmed: 23644091.

11. Fagundes RL, Mantica M, De Luca L, et al. Safety of single transseptal puncture for ablation of atrial fibrillation: retrospective study from a large cohort of patients. J Cardiovasc Electrophysiol. 2007; 18(12): 1277-1281, doi: 10.1111/j.1540-8167.2007.00958.x, indexed in Pubmed: 17883403.

12. Hammerstingl C, Lickfett L, Jeong KM, et al. Persistence of iatrogenic atrial septal defect after pulmonary vein isolation--an underestimated risk? Am Heart J. 2006; 152(2): 362.e1-362.e5, doi: 10.1016/j.ahj.2006.04.034, indexed in Pubmed: 16875923.

13. Rillig A, Meyerfeldt U, Birkemeyer R, et al. Persistent iatrogenic atrial septal defect after pulmonary vein isolation : incidence and clinical implications. J Interv Card Electrophysiol. 2008; 22(3): 177-181, doi: 10.1007/s10840-008-9257-7, indexed in Pubmed: 18461430.

14. Linhart M, Werner JT, Stöckigt F, et al. High rate of persistent iatrogenic atrial septal defect after single transseptal puncture for cryoballoon pulmonary vein isolation. J Interv Card Electrophysiol. 2018; 52(2): 141-148, doi: 10.1007/s10840-018-0352-0, indexed in Pubmed: 29574595.

15. Rillig A, Meyerfeldt U, Kunze M, et al. Persistent iatrogenic atrial septal defect after a single-puncture, double-transseptal approach for pulmonary vein isolation using a remote robotic navigation system: results from a prospective study. Europace. 2010; 12(3): 331-336, doi: 10.1093/europace/eup428, indexed in Pubmed: 20080903

16. Ha ACT, Wijeysundera HC, Birnie DH, et al. Real-world outcomes, complications, and cost of catheter-based ablation for atrial fibrillation: an update. Curr Opin Cardiol. 2017; 32(1): 47-52, doi: 10.1097/HCO.0000000000000348, indexed in Pubmed: 27755137.

17. Elayi CS, Darrat Y, Suffredini JM, et al. Sex differences in complications of catheter ablation for atrial fibrillation: results on 85,977 patients. J Interv Card Electrophysiol. 2018; 53(3): 333-339, doi: 10.1007/s10840-018-0416-1, indexed in Pubmed: 30062452.

18. Tripathi B, Arora S, Kumar V, et al. Temporal trends of inhospital complications associated with catheter ablation of atrial fibrillation in the United States: An update from Nationwide Inpatient Sample database (2011-2014). J Cardiovasc Electrophysiol. 2018; 29(5): 715-724, doi: 10.1111/jce.13471, indexed in Pubmed: 29478273.

19. De Greef Y, Ströker E, Schwagten B, et al. Complications of pulmonary vein isolation in atrial fibrillation: predictors and comparison between four different ablation techniques: Results from the MIddelheim PVI-registry. Europace. 2018; 20(8): 1279-1286, doi: 10.1093/europace/eux233, indexed in Pubmed: 29016870.

20. Kaiser DW, Fan J, Schmitt S, et al. Gender Differences in Clinical Outcomes after Catheter Ablation of Atrial Fibrillation. JACC Clin Electrophysiol. 2016; 2(6): 703-710, doi: 10.1016/j. jacep.2016.04.014, indexed in Pubmed: 29623299.

21. Zylla MM, Brachmann J, Lewalter T, et al. Sex-related outcome of atrial fibrillation ablation: Insights from the German Ablation Registry. Heart Rhythm. 2016; 13(9): 1837-1844, doi: 10.1016/j. hrthm.2016.06.005, indexed in Pubmed: 27289011.

22. Yang E, Ipek EG, Balouch M, et al. Factors impacting complication rates for catheter ablation of atrial fibrillation from 2003 to 2015. Europace. 2017; 19(2): 241-249, doi: 10.1093/europace/ euw178, indexed in Pubmed: 28172794. 